\title{
Effet des cycles sur le comportement d'un pieu isolé dans un sable sous chargement latéral
}

\author{
Luc Verdure*, Daniel Levacher**, Jacques Garnier*** \\ * Doctorant, L.G.C.N.S.N., Faculté des Sciences de Nantes \\ BP 92208 - 44322 Nantes cedex 3, 0251125555 , \\ luc.verdure@physique.univ-nantes.fr \\ ** Professeur, Centre de Géomorphologie, UMR 6143, Université de \\ Caen, 24 rue des Tilleuls, 14000 Caen, 0231565709 \\ daniel.levacher@geos.unicaen.fr \\ *** Chef de division, Reconnaissance et Mécanique des Sols, LCPC \\ Nantes, Route de Bouaye, BP 4129 - 44341 Bouguenais cedex, \\ 02408458 19, jacques.garnier@1cpc.fr
}

Résumé : De nombreux essais portant sur le comportement de pieux sous chargement latéral ont été menés depuis quelques années à la centrifugeuse géotechnique du L.C.P.C. Nantes. Cet article a pour objet de présenter une étude de faisabilité réalisée en 2000 concernant le comportement d'un pieu isolé sous chargement latéral quasi-statique par une force cyclique. Sont décrits succinctement le programme d'essais réalisé ainsi que les techniques d'expérimentation et d'analyse utilisées. On présente les résultats obtenus qui portent sur l'influence des cycles et notamment sur l'effet de leur amplitude sur la raideur globale du pieu, sur les déplacements en tête, sur le moment de flexion maximal et sur les courbes de réaction $P-y$.

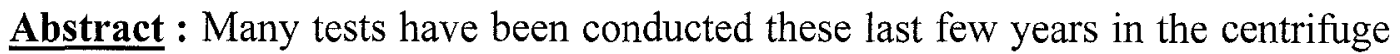
of the L.C.P.C. centre in Nantes in order to study the behaviour of laterally loaded piles. This paper concerns a feasibility study performed in the year 2000 on a single pile submitted to lateral cyclic loads. The experimental set up, measurements and data acquisition techniques are briefly given. Analyses are focused on the obtained results concerning the influence of cycles - mainly their amplitude - on the pile stiffness, on the top displacements, on the maximal bending moment and on the $P-y$ reaction curves.

\section{Introduction}

Les pieux isolés - pieux d'amarrage ou d'accostage - et les groupes de pieux de structures portuaires - quais - et offshore - plates-formes pétrolières - subissent le plus souvent des charges latérales cycliques pour des durées d'application variable dans le temps. Elles sont générées par la houle, les vagues, le mouvement de l'eau, le vent, la présence d'un navire. Les méthodes et règles destinées au calcul des pieux sous charges latérales statiques ont été certes aménagées pour la prise en compte de charges cycliques ou dynamiques mais elles restent inadaptées. 
Ces recommandations sont le plus souvent élaborées à partir d'essais in situ, (Kishida et al 1985 [5], Meimon et al 1986 [8], Little et al 1988 [6], Long et al 1994 [7]). La modélisation en centrifugeuse constitue une alternative intéressante aux essais en vraie grandeur non répétitifs et onéreux. C'est pourquoi des essais sur modèles réduits de pieu centrifugés, installés dans un sable sec et sous charges latérales cycliques ont été menés. Ils font suite à un vaste programme de travaux de recherche sur le comportement de pieux ou groupes de pieux sous charges latérales statiques initié au Laboratoire Central des Ponts et Chaussées de Nantes (LCPC).

La méthodologie expérimentale des essais réalisés en centrifugeuse sur un pieu isolé battu à $1 \mathrm{~g}$, dans un sable fin sec, soumis à des cycles, est présentée. L'effet des cycles, limités au nombre de 50 pour cette étude, est analysé à partir des courbes effort-déplacement et des courbes des moments fléchissants. La modification de l'allure des courbes de réaction constitue un résultat original. Cette modification traduit la dégradation de la réaction du sol en fonction $\mathrm{du}$ nombre de cycles et de la profondeur.

\section{Définition du programme expérimental}

\subsection{Cycle et taux de chargement}

Chaque cycle a été caractérisé par sa charge maximale $H_{\max }$ et son amplitude $\Delta H$. La charge varie donc entre $H_{\max }-\Delta H$ et $H_{\max }$. Lors de la définition du programme d'essais, il a été décidé de limiter l'étude des cycles à un seul paramètre qui est l'amplitude $\Delta H$. Le chargement est de type «one-way» (Chang et Whitman, 1988 [2]) : l'effort est toujours appliqué dans le même sens. Les valeurs de $\Delta H$ retenues sont : $0,2 . H_{\max }, 0,4 . H_{\max }, 0,6 . H_{\max }$ et $0,8 . H_{\max }$. Par la suite, les valeurs numériques dans cet article sont données, sauf mention expresse, en grandeur réelle (prototype). La valeur de la charge maximale choisie est de $960 \mathrm{kN}$, valeur de référence utilisée dans d'autres travaux de recherche sur le comportement de pieux isolés sous charge latérale statique (Remaud, 1999 [11]).

\subsection{Conteneur et désignation des essais}

Les essais ont été menés dans deux conteneurs différents. Chaque essai est désigné par le numéro du conteneur $(\mathrm{C} 1$ ou $\mathrm{C} 2)$ et par l'amplitude relative des cycles en pour-cent $(20,40,60$ ou 80$)$. Certains essais, parmi ceux effectués dans chaque conteneur, n'ont pas fourni de résultats exploitables. Nous avons pu analyser 2 essais du premier conteneur pour des pieux soumis à 15 cycles (essais $\mathrm{C} 1-40$ et $\mathrm{C} 1-60$ ) et 3 essais du deuxième conteneur dans lequel les pieux ont subi 50 cycles (essais C2-20, C2-40 et C2-80).

\section{Dispositif et techniques expérimentales}

Garnier, 1990 [4] décrit les caractéristiques de la centrifugeuse du LCPC. I1 rappelle aussi les conditions de similitude. Les essais de la présente étude ont été réalisés à $40 \mathrm{~g}$. 


\subsection{Reconstitution et caractérisation du massif de sable}

Le massif de sol est reconstitué par pluviation sèche de sable au moyen d'une trémie automatique mise au point par le LCPC. Ce dispositif produit un massif de sol très homogène avec une densité contrôlée (moins de $1 \%$ de variation). L'indice de densité choisi pour nos essais est de $I_{D}=95 \%$. Les propriétés physiques et la granulométrie du sable de Fontainebleau utilisé sont récapitulées dans les tableaux 1 et 2 .

Tableau 1 : Caractéristiques du sable de Fontainebleau utilisé pour l'étude.

Table 1: Characteristics of the Fontainebleau sand used for the study.

\begin{tabular}{|c|c|c|c|c|c|c|}
\hline \multicolumn{3}{|c|}{ Poids volumique $\left(\mathrm{kN} / \mathrm{m}^{3}\right)$} & \multicolumn{2}{c|}{ Indice des vides } & \multicolumn{2}{c|}{ Porosité } \\
\hline$\gamma_{\mathrm{s}}$ & $\gamma_{\mathrm{d} \max }$ & $\gamma_{\mathrm{d} \min }$ & $e_{\max }$ & $\mathrm{e}_{\min }$ & $\mathrm{n}_{\max }$ & $\mathrm{n}_{\min }$ \\
\hline 25,99 & 16,44 & 13,85 & 0,887 & 0,581 & 0,467 & 0,368 \\
\hline
\end{tabular}

Tableau 2 : Analyse granulométrique.

Table 2: Grain size distribution.

\begin{tabular}{|c|c|c|c|c|c|c|c|}
\hline Tamis $d(\mathrm{~nm})$ & 0,80 & 0,50 & 0,315 & 0,2 & 0,125 & 0,08 & 0,05 \\
\hline Passant $(\%)$ & 100 & 99,9 & 96,1 & 31,1 & 4,1 & 2,6 & 2,3 \\
\hline
\end{tabular}

D'autres moyens, plus qualitatifs, permettent de vérifier l'homogénéité du massif de sable du point de vue mécanique. Les comparaisons des courbes de battage (voir $\S 3.2$ ) et des profils pénétrométriques révèlent et attestent de la qualité du massif reconstitué. Un pénétromètre statique embarqué dans la nacelle de la centrifugeuse réalise ces profils.

\subsection{Caractéristiques, équipement et mise en place des pieux modèles}

Les pieux modèles ont déjà été utilisés lors d'essais effectués par Mezazigh (1995) [9] puis Remaud (1999) [11]. Ils simulent des pieux prototypes en acier de $15,2 \mathrm{~m}$ de longueur, $12 \mathrm{~m}$ de fiche, $0,72 \mathrm{~m}$ de diamètre et $16,5 \mathrm{~mm}$ d'épaisseur (échelle $1 / 40^{\text {ème }}$ ). Ils sont équipés de 20 couples de jauges de déformation répartis sur leur longueur, permettant de déterminer le moment fléchissant la fiche du pieu, de deux rotules en tête pour fixer les capteurs de déplacement et d'un trou permettant de passer le câble de traction pour l'application du chargement latéral. Une description plus complète de ces pieux est fournie par Remaud (1998) [10]. Les pieux sont mis en place par battage à $1 \mathrm{~g}$. Pour chaque conteneur, les 6 courbes de battage (nombre de coups par centimètre d'enfoncement du pieu) permettent de montrer qualitativement l'homogénéité du massif reconstitué.

\subsection{Dispositif et loi de chargement}

Pour charger le pieu latéralement, on utilise un servo-vérin asservi en force auquel on impose une courbe de chargement en fonction du temps. On mesure l'effort appliqué et deux déplacements en tête qui permettent de connaître la rotation en tête de pieu et le déplacement au point d'application de la charge. 
On cherche à appliquer un chargement quasi-statique. La montée en charge, de 0 à $H_{\max }=600 \mathrm{~N}$ (960 kN en grandeur prototype), est appelée "premier chargement". Chaque cycle est ensuite composé d'une descente puis d'une montée entre $H_{\max }$ $\Delta H$ et $H_{\max }$. La vitesse de chargement est de $10 \mathrm{~N} . \mathrm{s}^{-1}$ en grandeur modèle. Lors d'une montée ou d'une descente, on effectue 6 mesures. Ceci donne un nombre satisfaisant de points pour la détermination des courbes $P-y$, c'est-à-dire un point par valeur d'effort appliqué.

\section{Effet des cycles sur les déplacements en tête et les moments fléchissants}

\subsection{Analyse détaillée des courbes de chargement}

Les courbes effort-déplacement en tête, ou courbes de chargement, sont obtenues à partir des mesures. On peut constater, sur ces courbes de chargement, la présence d'une hystérésis au cours d'un cycle. A partir de ces courbes on peut calculer une "raideur globale" $K_{i}$ du système sol-pieu, à savoir le rapport entre un incrément de charge et un incrément de déplacement au point d'application de la charge. Cette raideur peut intéresser le concepteur puisqu'elle relie des paramètres directement utiles au dimensionnement. L'utilisation des courbes $P-y$ dans un calcul sert aussi à déterminer cette raideur. Nous avons choisi de calculer une raideur sécante comme le montre la figure 1.

\section{Courbe efforts-déplacements C1-40}

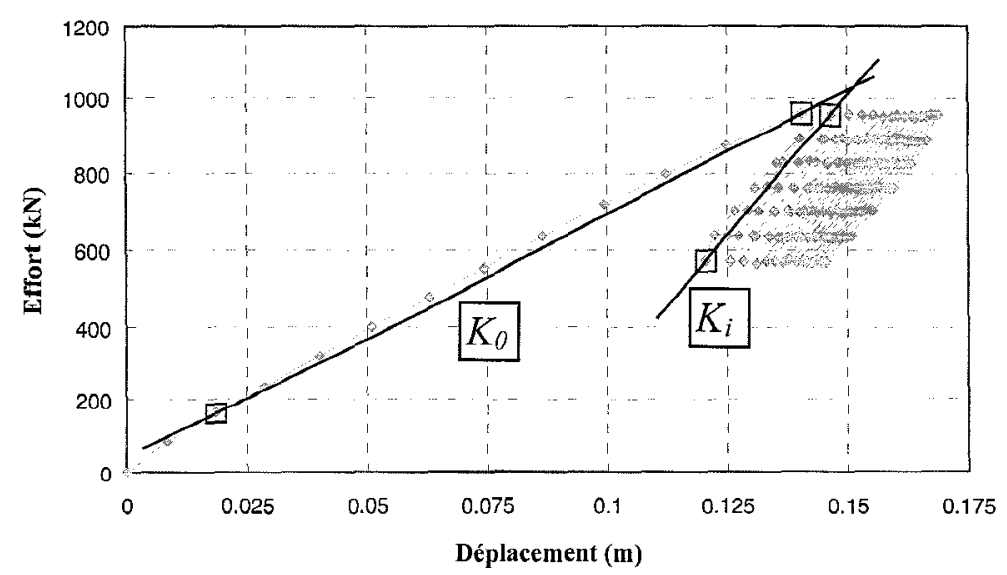

Figure $1:$ Méthode de détermination des raideurs globales du système sol-pieu.

Figure 1: Method of determination for the soil-pile system global stiffness.

Les raideurs $K_{i}$ ont été calculées, en valeurs prototype, pour les cinq essais exploitables. On s'intéresse à leur évolution en fonction du nombre de cycles. Cette évolution est exprimée sous la forme d'une raideur relative, rapport entre la raideur $K_{i}$ au cycle $i$ et la raideur $K_{l}$ au cycle 1 . Elle est montrée à la figure 2. Il apparait que le nombre de cycles a une influence sur les raideurs. De plus l'amplitude $\Delta H$ a tendance à accroître l'augmentation de la raideur globale apparente : de $5 \%$ pour $\Delta H=20 \%, 12 \%$ pour $\Delta H=40 \%$ et $25 \%$ pour $\Delta H=80 \%$, au bout de 50 cycles. 


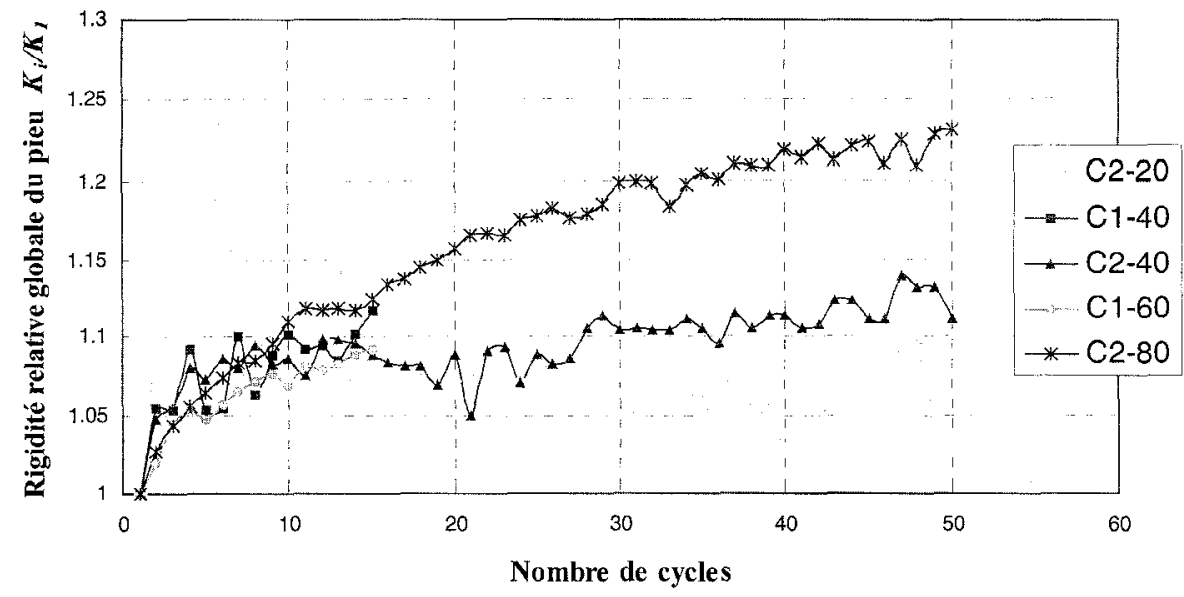

Figure 2 : Evolution de la raideur globale relative $K_{i} / K_{l}$ après le premier cycle en fonction du nombre de cycles.

Figure 2: Evolution of relative global relative stiffness versus number of cycles after the first cycle.

\subsection{Effet des cycles sur les déplacements en tête}

A partir des courbes effort-déplacement étudiées précédemment, on peut analyser l'évolution des déplacements au point d'application de la charge en fonction du nombre de cycles. Cette évolution est tracée pour le déplacement à $H_{\max }$. On compare l'évolution de ces déplacements pour différents essais en les exprimant sous la forme $y_{i} / y_{0}$ pour les rendre adimensionnels, $y_{i}$ étant le déplacement en tête à la charge maximale du cycle $i, y_{0}$ le déplacement en tête au maximum du premier chargement. Ces résultats sont représentés sur la figure 3.

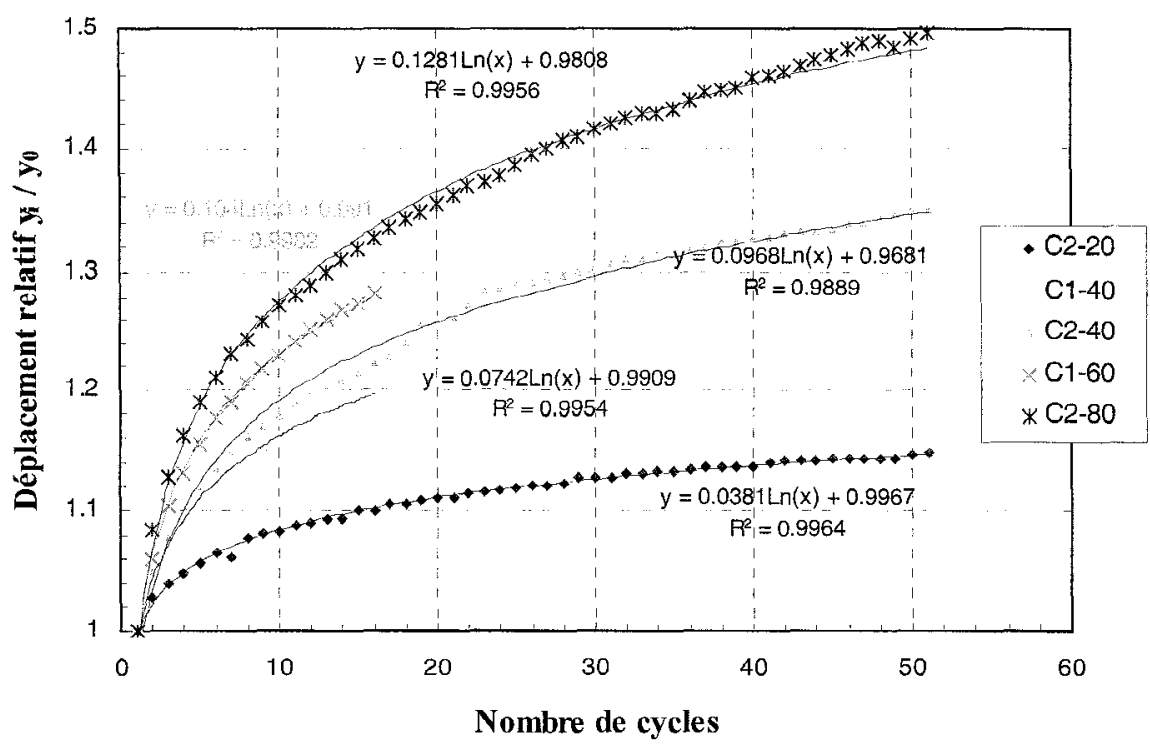

Figure 3 : Comparaison des évolutions des déplacements relatifs à $H_{\max }$ pour les différents pieux.

Figure 3 : Evolutions of relative displacements at $H_{\max }$ for different piles. 
On peut constater que ces évolutions, pour le nombre de cycles effectués, sont proches d'une loi logarithmique avec des coefficients de corrélation $R^{2}$ proches de 0,99 . L'amplitude des cycles accélère l'augmentation des déplacements relatifs. On peut exprimer le déplacement relatif sous la forme :

$$
\left\{\begin{array}{l}
\frac{y_{n}}{y_{0}}=1+A \cdot \log (n) \\
A=f(\Delta H)
\end{array}\right.
$$

avec $y_{0}$ le déplacement au maximum du premier chargement, $y_{n}$ le déplacement à la charge maximale du cycle $n$, $n$ le nombre de cycles, $A$ un coefficient adimensionnel dépendant de $\Delta H$.

Le coefficient $A$ caractérise le taux d'augmentation des déplacements relatifs. Si l'on essaie de modéliser $A(\Delta H)$ par une droite, qui nécessairement passe par l'origine, on obtient $A=1,8 \cdot 10^{-3} \cdot \Delta H$, avec $\Delta H$ en pour-cent de la charge maximale. Il est néanmoins indispensable de réaliser un plus grand nombre d'essais pour confirmer ou infirmer ce dernier modèle.

\subsection{Effet des cycles sur les courbes de moment fléchissant}

Le moment fléchissant maximal dans le pieu est une donnée intéressante pour le concepteur. Elle lui permet de savoir de combien il doit augmenter la résistance en flexion du pieu pour tenir compte de l'effet des cycles dans le dimensionnement.

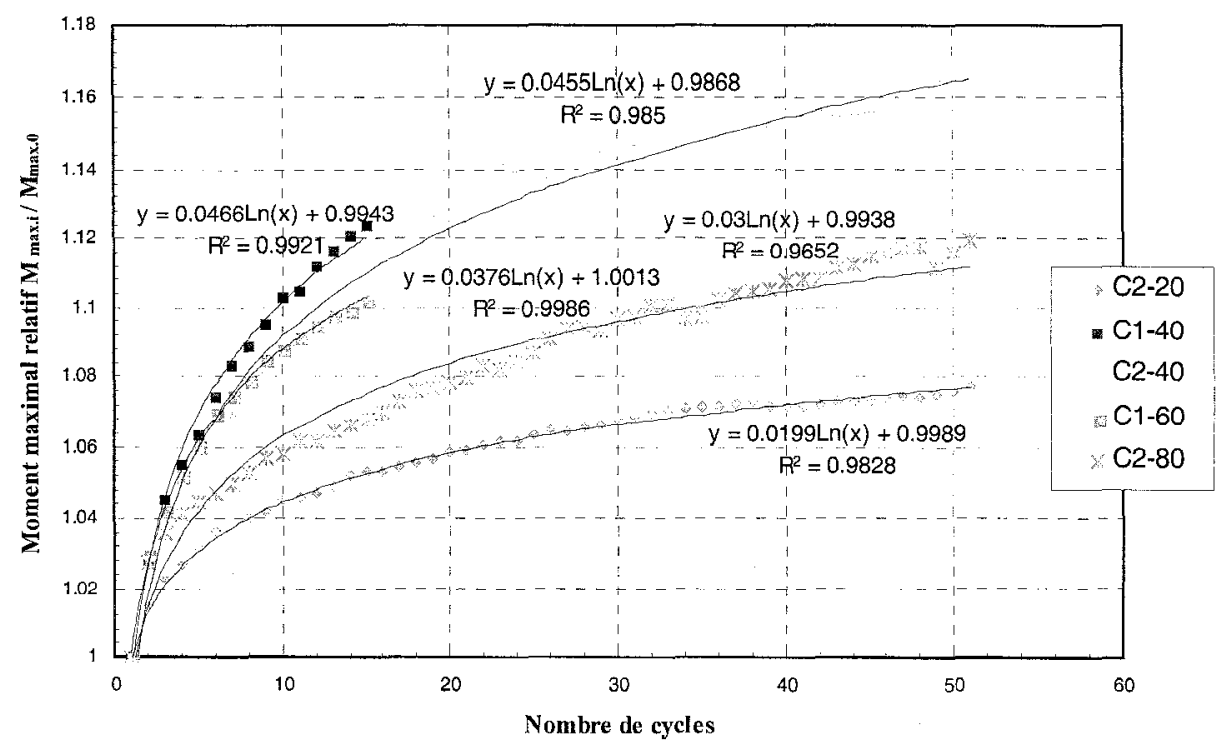

Figure 4 : Comparaison des moments maximaux à $H_{\max }$ relatifs pour différents pieux. Figure 4 : Evolutions of relative maximal bending moments at $H_{\text {max }}$ for different piles. 
L'étude de l'évolution de la valeur du moment maximal à la charge maximale en fonction du nombre de cycles a été menée de la même manière que celle des déplacements à charge maximale. Il s'agit de comparer un moment maximal $M_{\text {max. }}$ au cycle $i$ au moment maximal $M_{\max .0}$ à la fin du premier chargement. Les résultats sont représentés sur la figure 4.

Comme les déplacements, les moments maximaux à $H_{\max }$ ont une évolution proche d'une loi logarithmique, pour le nombre de cycles étudiés, comme le confirment les valeurs toutes supérieures à 0,96 des coefficients de corrélation $R^{2}$.

On peut proposer un modèle pour exprimer le moment maximal relatif en fonction du nombre de cycles :

$$
\frac{M_{\max . n}}{M_{\max .0}}=1+\mu \log (n)
$$

avec $M_{\max .0}$ le moment maximal à $H_{\max }$ pour le premier chargement,

$M_{\max . n}$ le moment maximal à $H_{\max }$ au cycle $n$,

$n$ le nombre de cycles,

$\mu$ un coefficient adimensionnel dépendant de $\Delta H$.

Le coefficient $\mu$, qui est, pour le concepteur, un coefficient amplificateur du moment maximal dans le pieu, caractérise le taux d'augmentation. Il dépend a priori du mode de mise en place et de la densité du sable, ainsi que de $\Delta H$. Les valeurs obtenues lors de nos essais sont comprises entre 0,02 et 0,05 . L'élaboration d'un modèle d'évolution de $\mu(\Delta H)$ nécessiterait des investigations supplémentaires.

\section{Analyse des courbes $P-y$}

\subsection{Construction des courbes $P-y$}

Les courbes de réaction $P-y$ sont déterminées à partir des mesures expérimentales au niveau de chaque couple de jauges de déformation. A partir de ces déformations, on détermine le profil du moment fléchissant $M_{f}(z)$.

Par double intégration de $M_{f}(z)$ on obtient le profil de déplacement $y(z)$. La valeur mesurée du déplacement au point d'application de la charge et la valeur de déplacement en pied fixée à 0 constituent les constantes d'intégration.

Par double dérivation de $M_{f}(z)$ on obtient le profil de réaction du sol $P(z)$ en N/m. Pour pouvoir dériver numériquement on effectue un lissage de $M_{f}(z)$ par spline quintique.

A partir des profils de déplacement et de réaction obtenus précédemment, on construit les courbes de réaction $P-y$. Chaque courbe $P-y$ est obtenue à une profondeur donnée. A chaque valeur de charge latérale $H_{i}$ correspond un couple $\left(P_{i}, y_{i}\right)$ pour une profondeur donnée. 
La totalité de cette procédure a été validée par Remaud (1999) [11] pour un chargement latéral statique. On admet qu'elle l'est aussi pour un chargement cyclique (quasi-statique).

\subsection{Etude de l'effet des cycles sur les courbes de réaction $P-y$}

L'étude de l'influence des cycles sur les courbes $P-y$ s'est avérée complexe. Nous nous sommes limités à l'étude de l'évolution des points $P-y$ à $H_{\max }$. Ces points seront par la suite notés $A_{i}$. De cette manière, il a été possible d'apporter quelques éléments de réponse au problème de l'influence des cycles sur les courbes de réaction. Les figures 5 et 6 représentent pour un pieu (C2-60) les courbes $P-y$ "statiques" ainsi que les points $A_{i}$ pour chaque cycle. Ces derniers ne forment pas une courbe de réaction mais dessinent uniquement l'évolution du point maximal de chaque courbe $P-y$ statique en fonction des cycles.

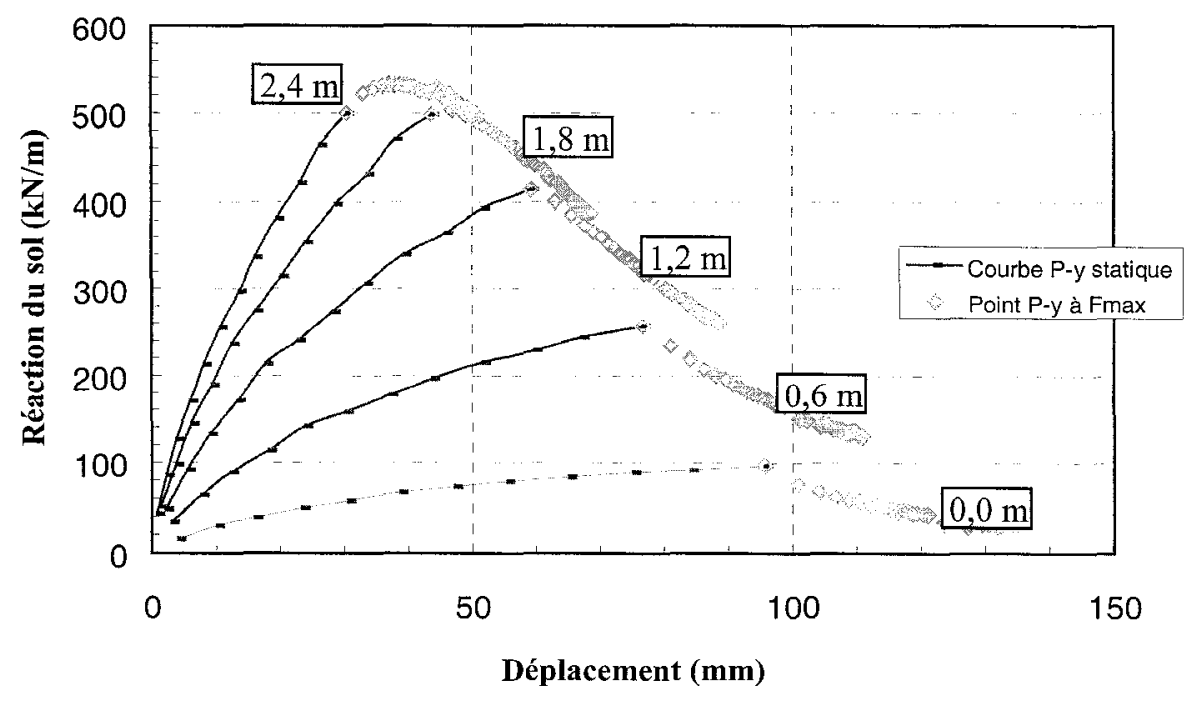

Figure 5 : Evolution des points $P_{i}$ pour les profondeurs de 0,0 à $2,4 \mathrm{~m}$.

Figure 5 : Evolution of $\mathrm{P}_{\mathrm{i}}$ points between 0,0 and $2,4 \mathrm{~m}$.

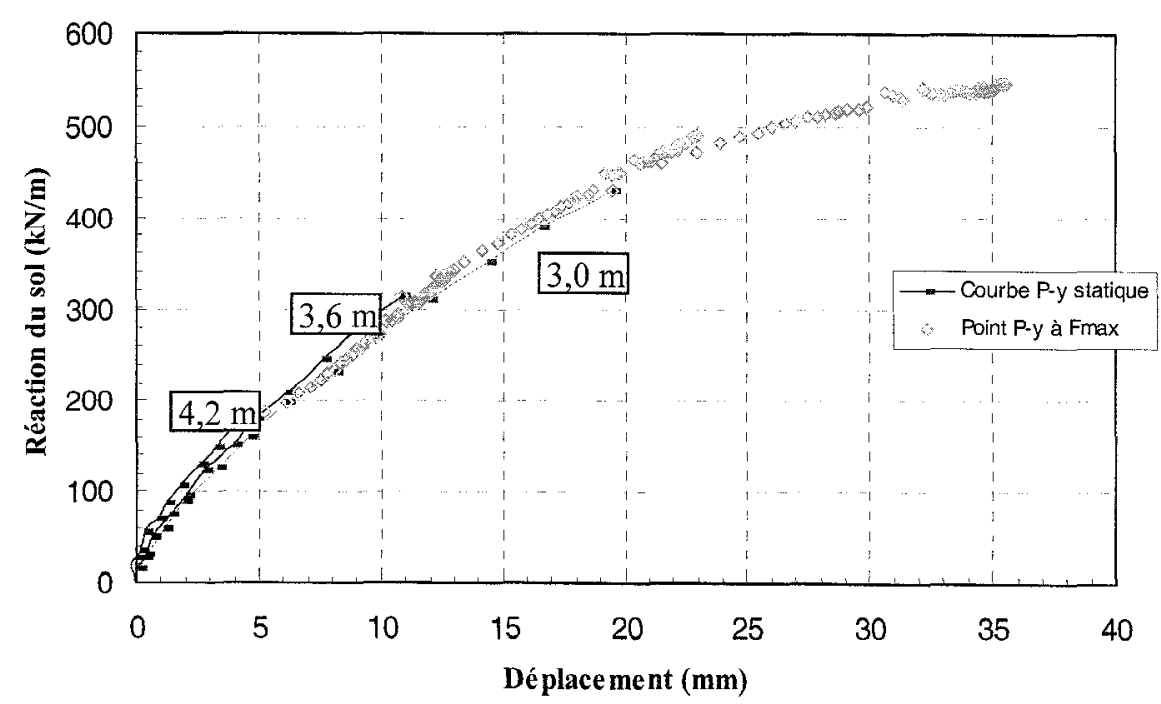

Figure 6 : Evolution des points $P_{i}$ pour les profondeurs de 3,0 à $4,2 \mathrm{~m}$.

Figure 6: Evolution of $\mathrm{P}_{\mathrm{i}}$ points between 3,0 and $4,2 \mathrm{~m}$. 
L'examen des figures 5 et 6 permet de voir l'évolution de la position des points $A_{i}$ en fonction des cycles. Dans tous les cas le déplacement augmente, alors que suivant la profondeur les réactions s'accroissent ou diminuent.

On constate sur ces courbes qu'à 15 ou 50 cycles, l'évolution relative de la réaction est d'autant plus importante que l'on est en profondeur. Cette évolution est négative pour de faibles profondeurs et crôt avec $z$. Elle change de signe, pour devenir une augmentation, à des profondeurs situées entre 1,8 et $3,0 \mathrm{~m}$. L'influence de l'amplitude $\Delta H$ est visible à ce niveau-là : plus $\Delta H$ est important, plus la profondeur du changement de signe est importante. En d'autres termes, l'amplitude des cycles affecte d'autant plus la capacité du sol à reprendre des efforts qu'elle est importante.

On peut considérer que, pour les courbes en surface $(0,0 \mathrm{~m}, 0,6 \mathrm{~m}$ et $1,2 \mathrm{~m})$, on a atteint lors de la phase statique le palier plastique comme le définit le Fascicule 62 : les déplacements évoluent alors que la réaction n'augmente presque plus. Ceci est surtout visible pour la courbe en surface, pour les deux autres on est proche du palier. Le fait que l'ordonnée des points $A_{i}$ diminue indiquerait donc que les cycles ont pour effet de diminuer la valeur en réaction du palier plastique. Ce résultat est en accord avec les recommandations A.P.I. (American Petroleum Institute, 1987 [1]) et D.N.V. (Det Norske Veritas, 1977 [3]) qui tiennent compte des cycles sur les courbes $P-y$ surtout en faible profondeur. Pour l'A.P.I. les corrections des courbes de réaction s'effectuent jusqu'à une profondeur relative $z / B=2,62$, pour D.N.V. jusqu'à $z / B=2$. Si l'on considère que dans notre cas les courbes $P-y$ sont affectées surtout jusqu'à $2,4 \mathrm{~m}$, d'après les figures 5 et 6 , on obtient une profondeur relative de 3,33 .

A l'inverse, pour des courbes plus profondes $(3,0 \mathrm{~m}, 3,6 \mathrm{~m}$ et 4,2 $\mathrm{m})$, l'enveloppe des points $A_{i}$ semble poursuivre la courbe $P-y$. Dans cette zone, le sol est toujours sollicité en pseudo-élasticité, les points $A_{i}$ se situent donc toujours sur la partie quasi-linéaire des courbes de réaction. Les cycles n'ont donc pas d'effet sur les courbes $P-y$. La diminution des réactions dans les parties du sol avoisinant la surface fait qu'en profondeur, les déplacements et les réactions sont plus importants. La reprise des efforts par le sol se décale vers les couches plus profondes.

Ces constatations amènent à penser que l'influence des cycles sur les courbes de réaction dépend du niveau de la sollicitation. Plus on est proche du palier plastique, plus l'ordonnée des points $A_{i}$ tend à diminuer. Il faudrait vérifier cette hypothèse pour les points proches du centre de rotation, zone située vers $4,0 \mathrm{~m}$ de profondeur, ce qui semble difficile étant donné que les déplacements et les réactions y sont faibles.

\section{Conclusions}

Cette campagne d'essais réalisée sur des pieux modèles installés dans un sable sec en centrifugeuse soumis à des chargements latéraux cycliques lents de type « one way » s'est avérée performante et prometteuse, (Verdure, 2000 [12]). En effet ces 
essais, par la nature même du chargement, constituaient une première investigation. La maîtrise de la technique expérimentale et la qualité des mesures permettent d'envisager des études paramétriques sur le comportement de pieux soumis à des charges latérales cycliques. Il serait intéressant notamment d'étudier l'influence de la charge maximale, du mode de mise en place, de la densité du sable et de l'effet de groupe.

\section{Références bibliographiques}

[1] American Petroleum Institute (A.P.I.) (1987), Recommended practice for planning, designing and constructing fixed offshore platforms, RP2A, Dallas, Texas, pp. 51-54.

[2] Chang C.S., Whitman R.V. (1988), Drained permanent deformation of sand due to cyclic loading, Journal of Geotechnical Engineering, ASCE, Vol. 96, $\mathrm{n}^{\circ} 5$, pp. 1605-1627.

[3] Det Norske Veritas (D.N.V.) (1977), Rules for the design construction and inspection of offshore structures, Appendix F, Foundations, reprint with corrections (1980), pp. 13-17.

[4] Garnier J. (1990), Etude sur modèles réduits centrifugés en génie civil, $1^{\text {ères }}$ Journées Génie Civil - Génie Côtier, Le Havre, pp. 101-119.

[5] Kishida, Suzuki, Nakai (1985), Behaviour of a pile under horizontal cyclic loading, Proc. Of the $11^{\text {th }}$ Inter. Conf. On Soil Mechanics and Foundation Engineering, San Francisco, Vol. 3, pp. 1413-1416.

[6] Little R.L., Briaud J.L. (1988), Full scale cyclic lateral load tests on six single piles in sand, pper GL-88-27, Geotechnical Div., Texas A \& M Univ., College Station, Texas, $30 \mathrm{p}$.

[7] Long J.H., Vanneste G. (1994), Effects of cyclic lateral loads on piles in sand, Journal of Geotechnical Engineering, ASCE, Vol. 120, $n^{\circ} 1$, January, pp. 225244.

[8] Meimon, Baguelin, Jézéquel (1986), Pile group behaviour under long time lateral monotonic and cyclic loading, Proc. Of the $3^{\text {rd }}$ Inter. Conf. On Numerical Methods in Offshore Piling, Nantes, France, May 21-22; Technip Editor, pp. 285-302.

[9] Mezazigh S. (1995), Etude expérimentale de pieux chargés latéralement : proximité d'un talus et effet de groupe, Thèse de doctorat, Université de Nantes.

[10] Remaud D., Garnier J., Frank R. (1998), Pieux sous charges latérales : étude de l'effet de groupe, $5^{\text {èmes }}$ Journées Nationales Génie Civil - Génie Côtier, Toulon.

[11] Remaud D. (1999), Pieux sous charges latérales: étude expérimentale de l'effet de groupe, Thèse de doctorat, Université de Nantes.

[12] Verdure L. (2000), Modélisation physique de pieux sous charge latérale cyclique dans du sable sec: étude de faisabilité, DEA Génie civil - Génie côtier, Université de Nantes, 74 p. 